\title{
La grammaire iconographique de la révolte en Syrie : Usages, techniques et supports
}

The Syrian Rebellion: a grammar of iconography

\section{Cécile Boëx}

\section{(2) OpenEdition}

12 Journals

\section{Édition électronique}

URL : http://journals.openedition.org/conflits/18789

DOI : $10.4000 /$ conflits. 18789

ISSN : $1777-5345$

Éditeur :

CECLS - Centre d'études sur les conflits - Liberté et sécurité, L'Harmattan

\section{Édition imprimée}

Date de publication : 31 décembre 2013

Pagination : 65-80

ISBN : 978-2-343-02618-3

ISSN : 1157-996X

Référence électronique

Cécile Boëx, "La grammaire iconographique de la révolte en Syrie : Usages, techniques et supports ", Cultures \& Conflits [En ligne], 91/92 | automne/hiver 2013, mis en ligne le 31 décembre 2014, consulté le 31 mars 2021. URL : http://journals.openedition.org/conflits/18789 ; DOI : https://doi.org/10.4000/ conflits. 18789 


\section{La grammaire iconographique de la révolte en Syrie Usages, techniques et supports}

\section{Cécile BOEX}

Cécile Boëx est Maître de conférences à l'EHESS et membre du CEIFR. Ses recherches portent sur les dimensions politiques des images en mouvement dans le monde arabe, qu'elles appartiennent au registre du cinéma ou qu'elles soient médiatisées par les nouvelles technologies d'information et de communication. Après un post-doc consacré à la grammaire filmique de la révolte en Syrie, elle s'intéresse plus particulièrement aux pratiques de témoignage par les images en contexte de conflit politique, notamment du point de vue des questions de la véridiction et des nouvelles modalités de fabrique $d u$ "martyre ".

T a révolte en Syrie, dont l'issue demeure toujours incertaine au moment où Lnous écrivons ces lignes, produit dans son sillage un corpus iconographique abondant et hétéroclite, imbriquant les espaces physiques des actions protestataires et l'espace virtuel d'Internet. Ces productions visuelles, particulièrement foisonnantes durant la première phase du mouvement caractérisée par le recours à des modes d'action pacifistes ${ }^{1}$, déploient divers registres graphiques et sémantiques dont l'objectif est à la fois d'informer, de sensibiliser, de persuader et de défier le régime. L'accessibilité des technologies ordinaires pour filmer les événements, mais aussi pour créer de nouvelles images numériques, contribue à façonner et à inventer une iconographie singulière et composite. Cette contribution propose une exploration des usages, des techniques et des supports de cette production visuelle dans un contexte où la mise en image de la révolte constitue un enjeu majeur. En effet, dès les premières manifestations, le régime tente de la faire passer pour une entreprise terroriste

1. Amorcé en mars 2011 à Deraa, au sud de la Syrie, le mouvement de révolte prend d'abord la forme de manifestations pacifiques qui s'étendent progressivement à d'autres régions du pays. Rapidement confronté à une répression de type militaire, le mouvement prend partiellement et graduellement une tournure violente à partir de l'été 2011, qui voit des officiers déserteurs établir l'Armée syrienne libre. À compter de février 2012, le bombardement à l'artillerie lourde de Homs puis des autres bastions de la révolte fait définitivement basculer le pays dans la guerre. Néanmoins, les actions protestataires pacifiques perdurent. 
et de dissimuler sa répression au moyen d'une campagne de désinformation et d'un embargo médiatique. Les dispositifs iconographiques produits par les manifestants et les activistes participent ainsi pleinement d'une lutte qui se joue également sur le terrain sémantique. De même, ces dispositifs s'articulent à des pratiques protestataires ${ }^{2}$ plurielles, modelées par des ressources, des contextes d'élaboration et de publicisation spécifiques. Sans prétendre à l'exhaustivité et dans l'impossibilité, pour l'heure, de procéder à une sociologie des faiseurs d'images opérant, pour la plupart, en vertu d'un principe d'anonymat, l'analyse ${ }^{3}$ se concentre sur les techniques et les contenus. Il s'agit, d'une part, d'éclairer l'émergence d'une nouvelle forme d'expression politique en Syrie qui puise son inspiration dans la caricature, le graff et l'affiche pour subvertir le discours et les symboles du régime. D'autre part, l'objectif est de mieux comprendre de quelles manières des acteurs protestataires s'approprient de nouvelles technologies de l'image pour relayer et mettre en forme leur lutte dans un contexte de répression extrême.

Les contours de cette production iconographique inédite sont esquissés à partir d'une réflexion sur leurs espaces d'élaboration (physiques ou numériques) afin de mieux comprendre comment ils interagissent, notamment par le truchement de la vidéo, et quels sont les supports, techniques et registres spécifiques qu'ils mobilisent. Dans un premier temps, les usages de l'iconographie sont questionnés du point de vue de leur ancrage concret au sein de modes d'action collectifs. Pancartes, graffitis et fresques sont observés plus spécifiquement à la lumière de quatre fonctions, souvent concomitantes : le cadrage ${ }^{4}$, la mise en scène visuelle des manifestations, l'acte de défi et la reconquête de l'espace public. Il s'agit également de voir comment les usages de la vidéo par les acteurs protestataires engendrent de nouveaux dispositifs iconographiques, agrégeant formes et messages à la performance. La focale de l'analyse se déplace ensuite sur les productions visuelles élaborées et diffusées au sein de l'espace virtuel : qu'est-ce qui caractérise cette iconographie dépourvue des contraintes propres aux espaces physiques des modes d'actions protestataires ? De quelles manières contribue-t-elle à la mise en récit de la révolte et à l'expression de ses mots d'ordre ? Pour commencer, ces questions sont abordées au prisme de deux collectifs particulièrement actifs dans ce domaine, spécialisés dans la réalisation d'affiches numériques. L'analyse permet ici de distinguer des stratégies communicationnelles différentes : l'une mobilisant des techniques et une imagerie propres aux médias de masse, l'autre s'inscrivant dans une démarche artistique. L'espace virtuel a par ailleurs donné lieu à un déchaînement symbolique contre la figure présidentielle, réappropriée et

2. Voir Siméant J., «Pratiques protestataires ", Genèses, 59-2, 2005, p. 2.

3. Elle s'appuie sur un corpus constitué à partir de vidéos de photographies collectées sur les réseaux sociaux et YouTube depuis le début de la révolution. Elle s'inscrit plus largement dans le cadre d'une expérience de terrain prolongée en Syrie qui nous a permis d'observer les dix premiers mois du mouvement de révolte.

4. Voir Benford R. D., Snow D. A., "Framing processes and social movements: an overview and assessment”, Annual Review of Sociology, 26, 2000, pp. 611-639. 
manipulée dans des photomontages. Axée plus spécifiquement sur une exploration des techniques numériques dans la production iconographique de la révolte, la dernière partie examine différents procédés visant au démantèlement du corps symbolique et mortel de l'homme de pouvoir.

\section{L'iconographie, support et source de modes d'action protestataires}

\section{Mises en scène visuelles des manifestations}

De façon générale, l'enjeu principal d'une manifestation réside dans l'imposition des manières d'interpréter ce qui s'y donne à voir et s'y exprime. Afin d'interpeller et de persuader, ce mode d'action déploie des dramaturgies 5 élaborées à partir de dispositifs de mise en scène propres. Dans ce sens, comme l'a montré David Kertzer, tout mouvement politique comporte un aspect rituel qui permet de créer des solidarités, de canaliser la perception des événements et de produire des légitimités ${ }^{6}$. En Syrie, les manifestations sont d'autant plus mises en scène qu'elles font l'objet d'un conflit d'interprétation avec le régime. De ce fait, les protestataires ont dû prendre en charge la publicisation de leurs actions, principalement au moyen de vidéos diffusées sur YouTube et les réseaux sociaux ${ }^{7}$. Aussi, la plupart des actions protestataires s'organisent en partie en fonction de leur mise en image. La coproduction audiovisuelle des actions protestataires met en jeu divers dispositifs iconographiques dont le but est d'authentifier le déroulement de l'action d'une part, et d'expliciter ses motifs pour sensibiliser l'opinion publique de l'autre.

Les vidéos documentant les manifestations sont en effet délivrées sans la médiation d'instances (journalistiques, juridiques ou morales) qui pourraient venir en attester la véracité. Conscients de ce problème, les preneurs d'images ont rapidement mis en place des dispositifs d'authentification visant à contextualiser les scènes filmées. C'est ainsi que chaque vidéo de manifestation commence par un écriteau tourné vers l'objectif de la caméra indiquant le lieu et la date. Ce dispositif mis en place dès les premiers mois de la révolte vise à coordonner les manifestations et à déjouer toute tentative de mise en doute de la temporalité de l'évènement. À ce recours pragmatique et informatif à l'écriture, s'ajoutent des usages plus sophistiqués qui impliquent des mises en scène de l'environnement des manifestations. Durant la première phase de la révolte, alors qu'il était encore possible d'organiser des rassemblements dans des localités relativement épargnées par la répression, des banderoles étaient accro-

5. Traïni C., «Dramaturgie des émotions, traces de sensibilités. Observer et comprendre des manifestations anti-corridas », Ethnographies.org, 21, 2010. Article en ligne, http://www.ethnographiques.org/Dramaturgie-des-emotions-traces (consulté le 22 août 2011).

6. Kertzer D., "Rituels et symbolisme politique dans les sociétés occidentales », L'Homme, 32121, 1992, pp. 79-89.

7. Voir Boëx C., «Montrer, dire et lutter par l'image. Les usages de la vidéo dans la révolution en Syrie », Vacarme, 61, 2012 (Article en linge, http://www.vacarme.org/article2198.html, consulté le 29 juillet 2013). 
chées sur les immeubles jouxtant la manifestation. Les activistes qui filment la manifestation prennent alors soin de montrer ces éléments ${ }^{8}$, permettant ainsi de mettre en exergue des mots d'ordre, de situer l'action et d'organiser sa cohérence visuelle. Les inscriptions, soigneusement calligraphiées, rappellent les techniques traditionnelles d'affichage publicitaire 9. Les manifestations sont de la sorte orientées vers l'impact qu'elles produiront sur l'opinion publique arabe mais aussi occidentale, certaines banderoles exposant des slogans en anglais. Elles constituent en cela un trait d'union inédit entre manifestations de rue et manifestations de papier ${ }^{10}$, dans la mesure où les protestataires jouent un rôle prépondérant dans la mise en image et l'interprétation de l'action collective, les journalistes n'ayant pas accès au terrain.

La mise en exergue de la dimension pacifiste des rassemblements protestataires participe d'une dénonciation de la répression et de la contestation de la légitimité du régime. De ce point de vue, la créativité et le registre de l'humour interviennent comme des dispositifs de sensibilisation ${ }^{11}$ qui mobilisent particulièrement l'iconographie. Ces dispositifs prennent appui sur des talents locaux. La petite ville de Kafranbel, par exemple, est désormais connue pour ses pancartes illustrées commentant de manière satyrique l'actualité de la révolte ( $c f$. document 1). Ces pancartes, directement inspirées du registre de la caricature politique ${ }^{12}$, ont rapidement acquis une renommée nationale puis internationale grâce à des photographies publiées sur les réseaux sociaux ${ }^{13}$. Des manifestations de soutien à la révolte en France ont d'ailleurs exposé des reproductions de ces pancartes. C'est une troupe de théâtre amateur qui a influencé l'identité iconographique des manifestations de la ville de Binnish, située dans la province d'Idlib. Lors de plusieurs manifestations, ses membres ont réalisé des fresques humaines, mettant en scène le nombre et la cohésion du groupe tout en adressant des messages au régime ${ }^{14}$ ou à la communauté

8. Comme on peut le voir par exemple sur cette vidéo, tournée le 24 février 2012 dans la petite ville de Hurrak, près de Deraa, en ligne : http://www.youtube.com/watch? $=0$ oaHV8BqNeCI (consultée le 29 juillet 2013). Sur les banderoles, on peut lire : «Les masques sont tombés », « Notre seule demande : un État démocratique ».

9. Avant l'arrivée récente des affiches publicitaires imprimées et des panneaux publicitaires modernes, les annonces d'évènements ou les enseignes commerciales étaient confiées à des calligraphes professionnels qui apposaient le message sur des banderoles. Malgré la multiplicité des supports publicitaires, cette pratique, moins coûteuse, a toujours cours. Voir Karl D. S., Zoghbi P., Arabic Graffiti (2e édition), Berlin, From here to fame, 2011.

10. Champagne P., Faire l'opinion, Paris, Éditions de Minuit, 1990.

11. Traïni C., Émotions...mobilisations!, Paris, Presses de Science Po, 2009.

12. Un genre déjà existant en Syrie avec des auteurs de renom comme Ali Ferzat. La caricature politique était tolérée par le régime dans la mesure où elle permettait une forme de critique euphémisée.

13. Une page Facebook dédiée à ces pancartes a été créée par des activistes de cette ville : http://www.facebook.com/kafar.nobol

14. Dans cette vidéo, tournée le 27 avril 2012, dans laquelle le texte apparaît en arabe et en anglais, la Syrie de Bachar al Assad est assimilée à l'oppression, la corruption, le despotisme et la destruction. En ligne : http://www.youtube.com/watch?v=kT8ml3msoYU\&feature=relmfu (consultée le 29 juillet 2013). 
internationale ${ }^{15}$. Ce groupe de comédiens amateurs a également imaginé une performance lors d'un rassemblement autour d'un puzzle géant d'une main formant le $\mathrm{V}$ de la victoire ( $c f$. document 2 ). Chaque partie de ce puzzle rappelle un massacre perpétré par le régime dans une ville (Hama, Houla, Qseir, etc.). Dans une vidéo qui présente un making-off de cette action ${ }^{16}$, les comédiens déclarent vouloir défier le régime en montrant la détermination des manifestants, malgré la répression. L'iconographie participe ainsi à une théâtralisation de l'action protestataire qui fait intervenir le corps, le mouvement et l'image dans des performances originales.

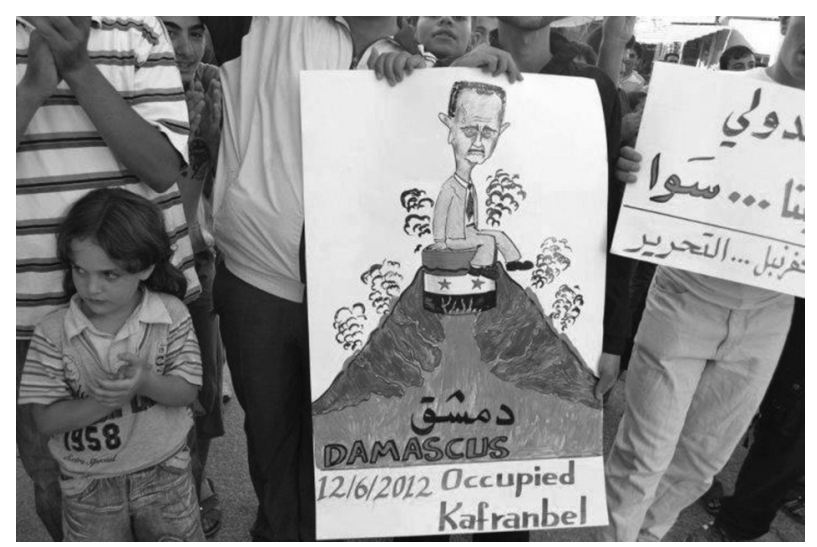

Document 1

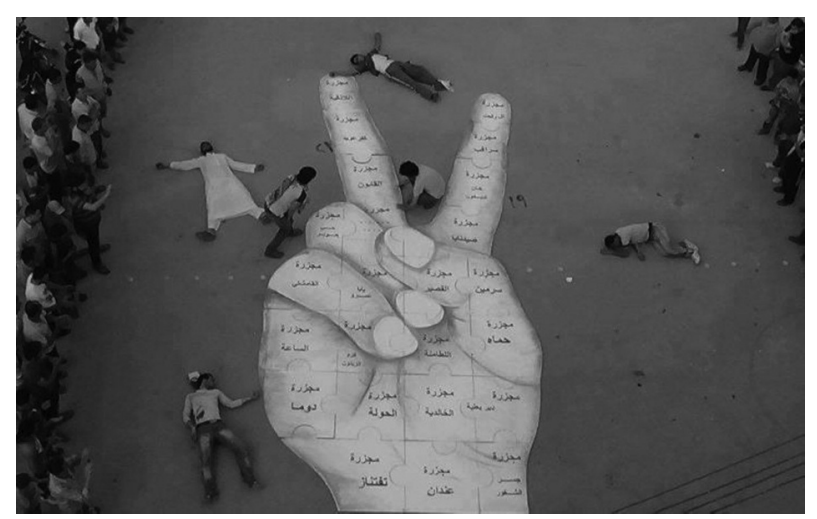

Document 2

15. Comme dans cette vidéo réalisée le 22 juin 2012, pendant le championnat européen de football. Les pancartes forment un terrain et un ballon circule entre les joueurs pour octroyer un point à l'équipe de la révolution contre celle du régime. Cette mise en scène dénonce l'indifférence de la communauté internationale. En ligne http://www.youtube.com/watch?v=P3VQLRiLfvA\&list=UUaQoUtyZWCMgGaoxTSAg1 OA\&index=306\&feature=plcp (consultée le 29 juillet 2013).

16. En ligne, http://www.youtube.com/watch?v=9hFjTksNwro\&list=UUaQoUtyZWCMg GaoxTSAg1OA\&index=333\&feature=plcp (consultée le 29 juillet 2013). 
La première manifestation à l'origine de la révolte a eu lieu à Deraa, suite à l'emprisonnement et à la torture subie par des écoliers qui avaient écrit sur un mur «Le peuple veut la liberté ». Depuis l'avènement du parti Baath en 1963, l'espace public a été confisqué au moyen d'un strict contrôle des formes d'expression. Il était alors impensable d'émettre publiquement ${ }^{17}$, même sous couvert de l'anonymat d'une inscription murale, une opinion hostile au régime. L'usage du graffiti protestataire dans le contexte de la révolte, bien qu'il n'engage pas le corps dans une confrontation directe avec les forces de sécurité, est une pratique à haut risque. À l'instar des manifestations, les inscriptions sur les murs expriment d'abord une opinion, enchâssée dans un acte de bravoure qui vient défier le régime. Elles reprennent d'ailleurs des slogans comme «Dégage ! », «Liberté », « Le peuple syrien est uni », « Nous nous prosternons seulement devant Dieu ». D'autres inscriptions investissent le registre injurieux : Bachar al-Assad et son père sont alors qualifiés d' "ânes ", de "chiens » ou de " traîtres ». Le risque inhérent à cette activité explique le caractère liminaire des inscriptions, pour la plupart écrites dans un geste hâtif afin d'esquiver les services de sécurité. De ce fait, l'écriture reste dominante et on trouve peu de dessin ou d'ornement, contrairement aux formes de street art qui ont fleuri lors des révoltes en Égypte 18 ou en Tunisie ${ }^{19}$. Certains talents locaux se sont néanmoins distingués par l'originalité de leurs productions, comme dans le village de al-Qusayr, près de Homs, où un artiste a réalisé des fresques murales ( $c f$. document 3 ) ou à Saraqeb, dont les murs sont connus pour leurs poèmes protestataires ornés de motifs floraux. Hormis ces formes singulières locales, l'activisme graphique ne tend pas vers une individuation des pratiques, supposant la mise en exergue d'un «auteur». On observe au contraire une uniformisation des inscriptions qui rend compte d'une pratique axée sur l'efficacité du message plutôt que sur une recherche formelle.

17. Des formes de critiques euphémisées existaient néanmoins, notamment dans le domaine de la production artistique et plus particulièrement du cinéma. Voir Boëx C., La contestation médiatisée par le monde de l'art en contexte autoritaire : l'expérience cinématographique syrienne au sein de l'Organisme général du cinéma (1964-2010), thèse de doctorat, IEP Aixen-Provence, 2011.

18. Sur ce phénomène, voir : Hamdy B., Karl D. S., Eltahawy M., Walls of Freedom: Street Art of the Egyptian Revolution, Berlin, From here to fame, 2013 ; Abaza M., "The Dramaturgy of Street Corner”, Jadaliyya, janvier 2013 (en ligne, http://www.jadaliyya.com/pages/index/9724/the-dramaturgy-of-a-street-corner (consulté le 29 juillet 2013), ainsi que Ali A., “Alexandria Re-Imagined: The Revolution Through Art”, Jadaliyya, janvier 2013 (en ligne : http://www.jadaliyya.com/pages/index/9760/alexandriare-imagined_the-revolution-through-art, consulté le 29 juillet 2013).

19. Voir par exemple : «La revanche du graffiti tunisien », Le Monde, 2 septembre 2011 ou « La révolution tunisienne s'affiche sur les murs à grand renfort de graffitis », France 24, 27 mai 2011 (en ligne, http://observers.france24.com/fr/content/20110527-revolution-tunisiennebombe-murs-pays-graffitis-tunis-art-urbain-Ahl-El-Kahf, consulté le 29 juillet 2013). 


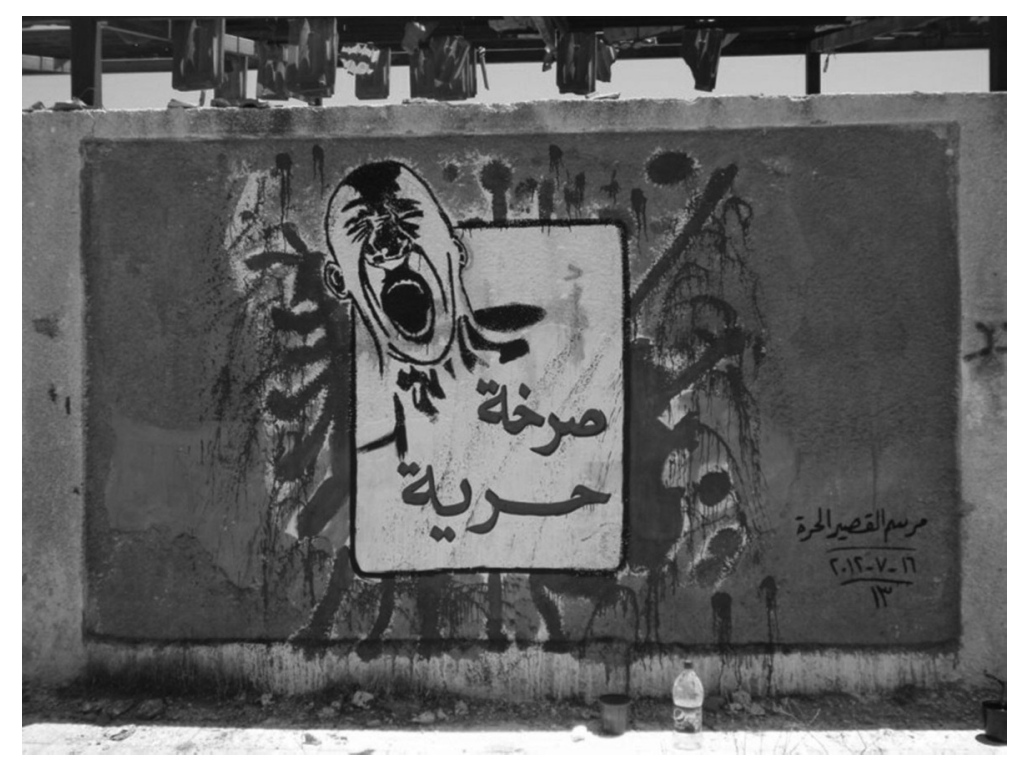

Document 3

Bien que les graffitis soient peu stylisés, cette forme d'activisme fait l'objet d'une organisation spécifique. Celle-ci s'est plus particulièrement répandue chez les jeunes, constitués en collectifs dans certaines villes comme à Damas, Tell, Soueida ou Qamichli. Leurs actions s'appuient sur l'enregistrement vidéo et les réseaux sociaux, principalement YouTube et Facebook. L'usage de ces technologies de mise en image et de publicisation génère une nouvelle forme d'activisme graphique, où les conditions de l'inscription du message importent tout autant que le message lui-même. Par ailleurs, la vidéo confère une visibilité et une longévité aux graffitis, souvent effacés le jour même par les forces de sécurité. Les graffeurs ont ainsi pris l'habitude de filmer leurs actions, tout en dissimulant leurs visages. La vidéo permet de contextualiser l'action et de rendre compte de la tension dans laquelle celle-ci s'effectue ${ }^{20}$. Associé à la captation audiovisuelle, le graffiti devient une performance où l'héroïsme de l'acte subversif en contexte hautement répressif est donné à voir. La diffusion de photographies où les graffeurs se mettent en scène contribue aussi à cette célébration de la bravoure, nécessairement anonyme. La mise en image et les réseaux sociaux permettent par ailleurs une diffusion des savoir-faire. De cette manière, la technique du pochoir, largement répandue chez les graffeurs égyptiens lors de la révolte, a pu inspirer certains activistes en Syrie. Celle-ci a notamment été reprise pour rendre hommage aux martyrs. Internet favorise également une coordination des actions et l'organisation des campagnes de graffitis. C'est le rôle de la page Facebook « Freedom

20. Comme on peut le voir sur cette vidéo tournée à Damas en septembre 2012, en ligne : http://www.youtube.com/watch?v=7hRvR9YOwrk\&feature=youtu.be (consultée le 29 juillet 2013). 
Graffiti Week Syria 21 »créée par un collectif d'activistes syriens ${ }^{22}$, sur le modèle de la page égyptienne «Mad Graffiti Week 23 ». En plus de centraliser les vidéos réalisées par les différents groupes de graffeurs dans le pays, permettant un archivage des graffitis, elle produit des vidéos pédagogiques expliquant certaines techniques ${ }^{24}$.

Si cette nouvelle forme d'activisme utilise l'espace virtuel pour se mettre en scène, diffuser et coordonner ses actions, son objectif premier est d'inscrire son empreinte dans des espaces concrets et d'agir sur leur perception. À cet effet, certains graffeurs détournent des éléments de l'environnement urbain pour mettre en forme leurs messages. De nombreuses inscriptions mettent en scène des bennes à ordure sur lesquelles on peut lire : "Bachar al-Assad est tombé ». Dans le même esprit, des dessins réalisés au pochoir permettent d'imprimer le visage de Bachar al-Assad sur des trottoirs où il est indiqué : «Marchez ici ». Le graffiti se conçoit en interaction avec l'environnement urbain et ses usages. L'activisme graphique participe ainsi d'une réappropriation physique et symbolique de l'espace public de manière non violente. Cette lutte symbolique fait d'ailleurs l'objet d'une véritable stratégie, observable dans les choix de certains sites privilégiés par les graffeurs, comme les écoles ou les centres culturels, relais traditionnels de l'idéologie baathiste. La réappropriation visuelle de ces bâtiments s'effectue aussi par la reproduction, à la bombe ou à la peinture, du drapeau de la révolution ${ }^{25}$, autre élément venant invalider et remplacer une symbolique liée au régime. S’inscrivant dans un registre pacifiste, ces actions peuvent néanmoins exprimer de la sympathie pour la lutte armée, comme en témoignent les insignes de l'armée libre réalisées à la peinture ou au pochoir dans différentes villes. Les murs sont ainsi devenus de véritables enjeux d'une lutte dont les coups échangés (graffitis immédiatement masqués à la bombe par les services de sécurité puis réapparaissant à côté) restent visibles et constituent autant de preuves de la détermination des protestataires ( $c f$. document 4). Lorsqu'une ville est libérée, l'une des premières actions des comités locaux de coordination est d'inscrire cette victoire dans le paysage urbain. Sur une vidéo tournée à Yabroud en octobre $2012^{26}$, la réorganisation du quotidien selon ce nouveau statut est signifiée et s'effectue en partie par le remodelage graphique et chromatique de l'espace public.

21. En ligne : http://www.facebook.com/MAD.GRAFFiTi.Week.SYRiaa?ref=ts\&fref=ts (consultée le 29 juillet 2013).

22. Nour Hatem Zahra était l'un des membres fondateurs. Grapheur de 23 ans agissant dans la ville de Damas et sa banlieue, il a été tué par les forces de sécurité en avril 2012.

23. En ligne : http://www.facebook.com/MAD.GRAFFiTi.WEEK (consultée le 29 juillet 2013).

24. Voir par exemple cette vidéo qui présente la technique du pochoir, en ligne : https://www.youtube.com/watch?v=1D7dj7GHuYI (consultée le 29 juillet 2013).

25. Ce drapeau est à l'origine celui adopté en 1946 lors de l'indépendance, puis abandonné à la création de la République arabe unie en 1961. Contrairement au drapeau officiel, les trois couleurs panarabes (le rouge, le noir et le vert) sont inversées et il comporte trois étoiles au lieu de deux. 


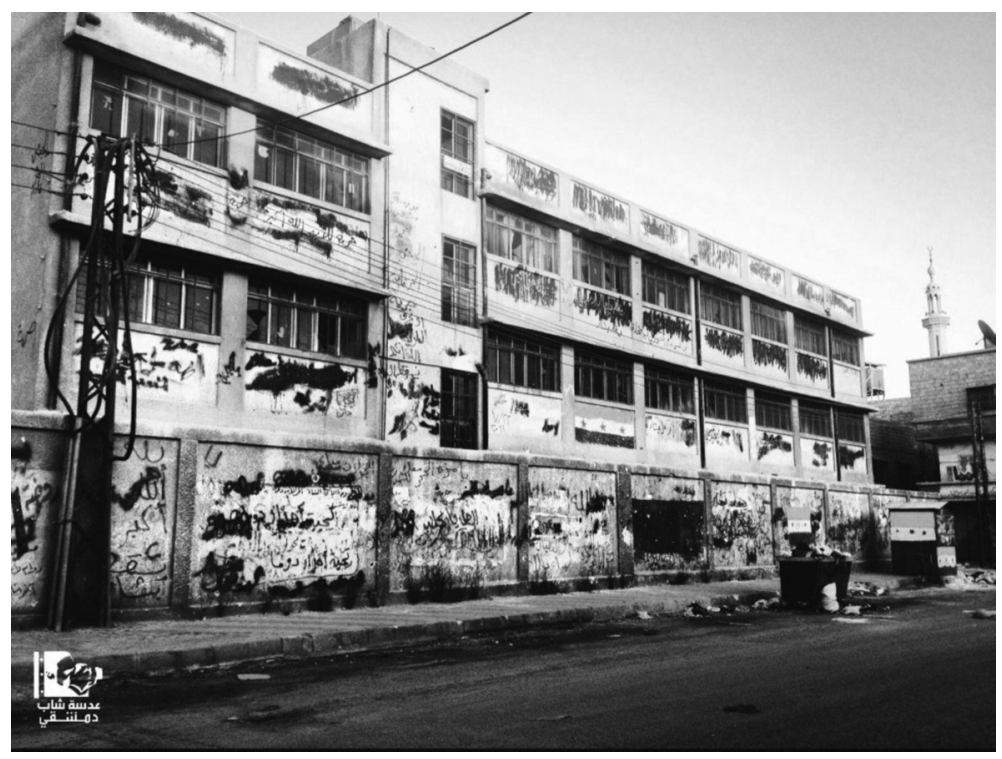

Document 4

\section{Techniques et registres de l'iconographie protestataire numérique}

\section{Entre information et création}

La production iconographique de la révolte se déploie également abondamment dans l'espace virtuel, donnant lieu à de nouvelles formes et à de nouveaux registres communicationnels. Le recours à Internet permet un activisme moins risqué et transnational. Parmi le foisonnement des images circulant sur les réseaux sociaux (sites d'information, blogs, pages Facebook de collectifs de designers ou d'artistes), les affiches numériques, réalisées par des professionnels ou des amateurs, constituent des supports privilégiés pour subvertir la propagande officielle, exprimer un point de vue, donner une consistance visuelle à des évènements marquants ou sensibiliser l'opinion publique sur l'ampleur de la répression. Cette forme de communication protestataire puise dans un vaste répertoire iconique qui mobilise aussi bien des images politiques issues du contexte local que des images renvoyant à un imaginaire révolutionnaire universel. Sur le plan formel, on observe une prégnance du visuel sur l'écrit. Contrairement à d'autres types d'iconographies politiques où l'image aurait plutôt une fonction emphatique ${ }^{27}$, venant illustrer le texte, le corpus de la trentaine d'affiches récoltées sur la toile fait d'abord parler l'image.

26. En ligne : http://www.youtube.com/watch?feature=player_embedded\&v=vr3fLh6KosA\#! (consultée le 29 juillet 2013).

27. Voir Memmi D., Du Récit en politique. L'affiche électorale italienne, Paris, Presses de Science Po, 1986. 
L'activisme iconographique sur Internet a engendré une véritable professionnalisation, avec la formation de collectifs. Elle donne lieu à une effervescence créative dont il est difficile de systématiser les techniques tant elles sont éclectiques. On observe néanmoins deux grandes tendances. Empruntant au registre visuel des médias de masse (publicité, journaux télévisés, cinéma), la première est conçue comme un véritable outil de mobilisation et de coordination. La seconde s'inscrit davantage dans une démarche artistique. Le collectif le plus représentatif du premier courant est l'« Équipe multimédia » qui publie sa production sur une page Facebook 28 comptant près de 95000 abonnés en juillet 2013. Ce collectif est particulièrement actif avec plus de 300 affiches, flyers, photographies et dessins mis en ligne depuis sa création en mars 2011. Fonctionnant sur le principe de l'anonymat, chaque réalisation est signée par le collectif. En plus de dénoncer la propagande du régime, la production de l'Équipe multimédia prend en charge plusieurs aspects de la communication visuelle du mouvement de révolte. Des affiches sont ainsi dédiées aux « martyrs » et, chaque vendredi, un flyer illustré, associé à un slogan spécifique commun à toutes les manifestations ${ }^{29}$, est diffusé. L'action du collectif se veut également concrète avec des campagnes de désobéissance civile incitant la population à faire grève par exemple. Une autre campagne expose, à travers le dessin et le texte, différentes consignes pour coopérer avec l'armée libre 30, recruter des jeunes hommes sachant manier des armes ou mener une préparation militaire personnelle, conçue comme un moyen d'autodéfense ${ }^{31}$.

La production iconographique du collectif « Le peuple syrien sait où il va » mobilise un registre différent. Constitué par une quinzaine d'activistes résidant pour la plupart à l'extérieur de la Syrie, sa production s'inscrit au sein d'une activité de communication plus vaste, diffusée sur un blog et sur une page Facebook. Ce groupe propose un point de vue distancié et analytique sur les évènements, en accordant une place privilégiée aux expressions artistiques. Il relaye ainsi des articles de fond de la presse arabe ainsi que des affiches et des vidéos produites par des artistes. Depuis le début de la révolte, ce collectif a réalisé près de 200 affiches, visibles sur la galerie photo de leur compte Flickr ${ }^{32}$. Le langage visuel mobilisé n'est pas forcément en relation directe avec les images et les événements propres à la révolte, contrairement à la production iconographique de l'Équipe multimédia. Celui-ci s'élabore principalement à partir d'un recyclage original de références iconographiques politiques universelles. Le graphisme, les couleurs et certains motifs comme le

28. En ligne : http://www.facebook.com/rev.multimediateam?ref=ts\&fref=ts (consultée le 29 juillet 2013).

29. Cette uniformisation nationale du mot d'ordre du vendredi, jour principal de manifestation, permet de coordonner l'action et d'en attester la date. Ce slogan fait l'objet d'un vote préalable organisé sur la page Facebook «The Syrian Revolution 2011», l'une des pages consacrées au mouvement les plus populaires.

30. Soigner et cacher les blessés et fournir des informations sur les positions ennemies.

31. L'affiche conseille ainsi un entraînement aux arts martiaux et préconise de se procurer des armes légères.

32. En ligne : http://www.flickr.com/photos/3aref/?saved=1 (consultée le 29 juillet 2013). 
point levé, les médaillons entourés d'épis de blé, le petit livre rouge, le hautparleur ou les rayons, sont directement issus de l'iconographie communiste et socialiste. On observe également une hérö̈sation de l'identité collective ${ }^{33}$, représentée sous les traits du peuple, des femmes, des étudiants et des enfants ${ }^{34}$. À contre-courant de la rhétorique du régime qui attise la haine entre les communautés religieuses, le collectif met l'accent sur le caractère populaire, pluraliste et non violent de la révolte. Sa stratégie communicationnelle est clairement orientée vers une volonté d'universaliser le combat du peuple syrien en l'inscrivant dans l'historicité de luttes plus anciennes. Ainsi, une affiche invite « La femme syrienne » à se révolter contre " tous les pouvoirs » ( $c f$. document 5$)$ en référence au combat féministe. Ce visuel est d'ailleurs une version remaniée d'une affiche d'un groupe socialiste féministe belge ${ }^{35}$. De même, bien que l'arabe demeure la langue dominante et fasse l'objet d'un raffinement et d'une recherche calligraphique, certaines affiches contiennent des messages en anglais, en français, en espagnol ou en kurde. L'identité visuelle et l'esthétique singulière de la production iconographique de ce collectif lui ont valu une reconnaissance dans le monde de l'art européen, avec des expositions en Angleterre ou en Italie.

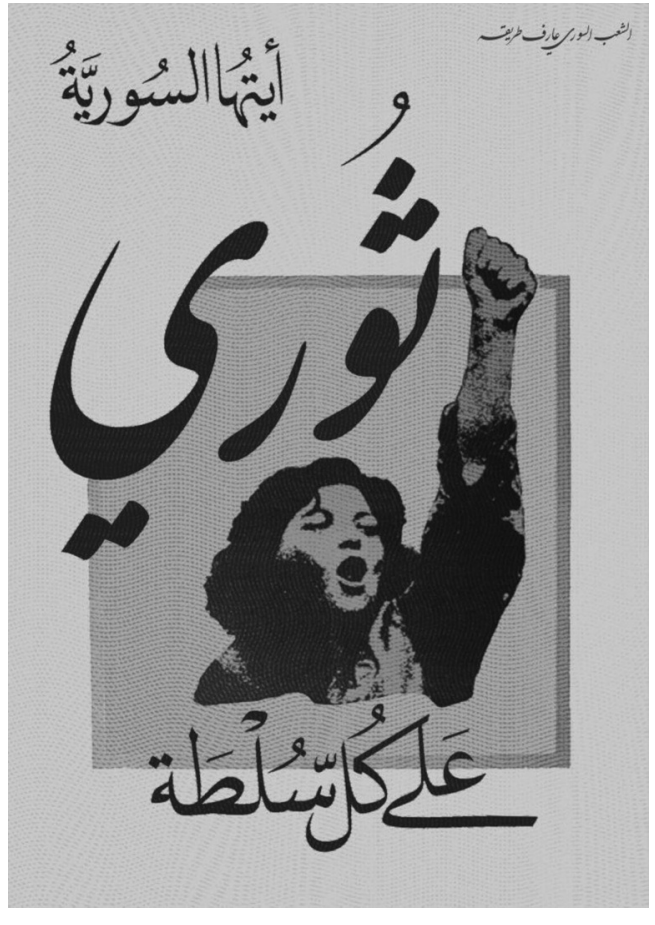

Document 5

33. Buton P., «L'adieu aux armes ? L'iconographie communiste française et italienne depuis la Libération ", Vingtième siècle. Revue d'histoire, 80, 2003, pp. 43-54.

34. Cette catégorie est plus particulièrement spécifique à l'imagerie maoïste.

35. Il s'agit du Groep Rooie Vrouwen (GROV, Groupe des femmes rouges) créé dans les années 1960 qui faisait partie du mouvement plus vaste FemSoc, comprenant les militantes néerlandaises du groupe Dolle Mina. L'affiche originale est publiée sur le blog « the feminist poster project », en ligne : http://feministposterproject.wordpress.com/ (consultée le 29 juillet 2013). 
Au début de la révolte, certaines manifestations ont donné lieu à des actes iconoclastes contre les objets du culte présidentiel. Particulièrement développé durant le régime de Hafez al Assad ${ }^{36}$, celui-ci s'est prolongé avec l'arrivée de son fils au pouvoir en 2000 , dans une version plus moderne. La détérioration physique des figures symboliques du régime par la force (le déboulonnage de statues), le feu (la destruction d'affiches) ou la matière (dissimulation ou ajouts de traits caricaturaux aux portraits) apparaît dans de nombreuses vidéos et photographies largement diffusées sur Internet et les médias internationaux. Cette suppression symbolique de deux figures centrales du régime, visant à effacer leur empreinte dans l'espace public et dans l'histoire, a également donné lieu à une production iconographique numérique originale, dirigée plus spécifiquement contre Bachar al Assad. Des activistes s'emparent de son image et de sa personne pour lui faire subir toutes sortes de métamorphoses avilissantes. L'accessibilité des logiciels de création graphique et photographique permet de le déformer, de le travestir, de le désintégrer, grâce aux différentes techniques d'incrustation, de montage et de trucage. Dans certains visuels, la figure présidentielle disparaît, remplacée par les héros anonymes et ordinaires de la révolte. Cette violence symbolique médiatisée par l'outil numérique puise dans les techniques traditionnelles de la caricature, axées sur les déformations. Elle en invente également de nouvelles, permettant des juxtapositions et des déplacements de sens inédits et inattendus.

Au sein du corpus iconographique ciblant la figure présidentielle, on observe, si l'on procède par analogie avec l'analyse d'Ernest Kantorowicz ${ }^{37}$, un processus de dissociation entre le corps terrestre et mortel du président et son corps politique et immortel. La transsubstantiation de l'individu à l'homme de pouvoir réalisée par les différentes formes de sa représentation officielle ${ }^{38}$ se trouve ici inversée : Bachar al Assad est destitué de son corps glorieux, incarnation de la fonction politique, pour être renvoyé à la corporéité d'un individu auquel on attribue la responsabilité des crimes commis contre les protestataires ( $c f$. document 6$)$. Cette mise à nue dénonciatrice s'articule à une opposition public/privé, endroit/envers, apparence/vérité. Le

36. Le culte de la personnalité du président s'est construit au fil des évènements politiques : la «victoire » de la guerre israélo-arabe d'octobre 1973, la décision impopulaire d'Assad d'intervenir militairement au Liban en 1976 contre les forces progressistes palestiniennes et l'écrasement de la révolte en 1982 à Hama. C'est suite à la crise cardiaque de Hafez al Assad et à la tentative de coup d'État par son frère Rifa'at al Assad qu'une imagerie sainte rendant le leader éternel et immortel apparaît et qu'on assiste à la construction de monuments et de statues à sa gloire. Les représentations du président sont multiples : il est à la fois le père, le combattant victorieux capable de protéger la nation arabe et de restaurer sa dignité ainsi que le patron de toutes les corporations professionnelles. Voir Wedeen L., Ambiguities of Domination, Politics, Rhetoric and Symbols in Contemporary Syria, Chicago, The University of Chicago Press, 1999.

37. Kantorowicz E., Les Deux corps du roi, Paris, Gallimard, 1989 [1957].

38. Un processus analysé à partir des portraits de Louis XIV par Marin L. dans Le Portrait du roi, Paris, Éditions de Minuit, 1981. 


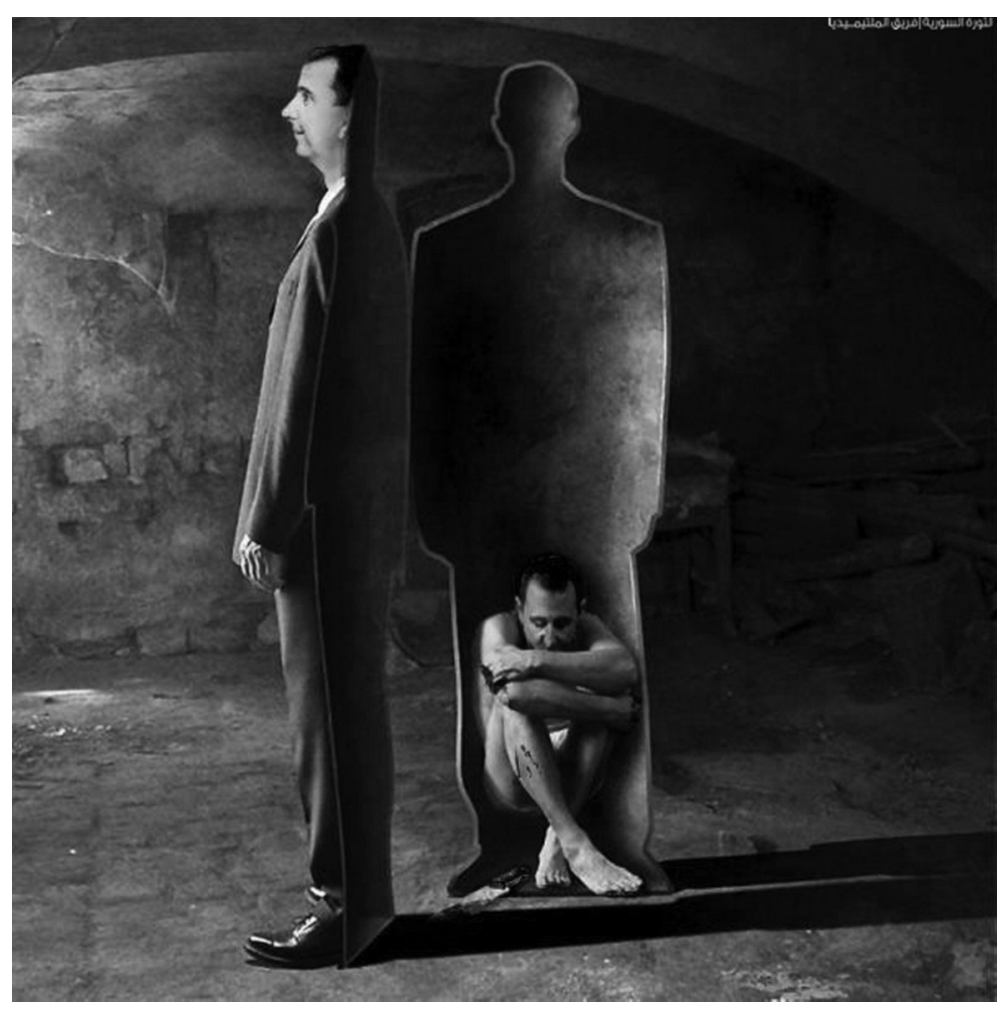

Document 6

corps de chair est exhibé et souillé (par le sang ou la boue). Il est également placé dans des lieux répugnants comme sur un montage photographique montrant Bachar al Assad dans un égout, le haut du corps et le visage maculés d'immondices. Les techniques de trucage permettent également de remodeler ce corps. Là encore, c'est sa dimension ordinaire qui est saisie dans son individualité. Les images ayant recours à ce procédé mobilisent le registre du grotesque, lequel, caractérisé par le monstrueux et la difformité, « abolit les frontières du corps ${ }^{39}$ ». L'animalisation 40 du président, construite sur une ressemblance physique ou psychologique, émerge comme un motif récurrent. Les métaphores animalières dont il fait l'objet subvertissent la symbolique du lion associée au nom Assad (qui signifie «lion» en arabe), fréquemment utilisée dans le discours et l'iconographie pro-régime ${ }^{41}$. En écho avec certains slogans

39. Silhouette M., « Présentation », Sociétés E Représentations, 10, 2000, pp. 4-6.

40. C'est un procédé classique de la caricature qu'on rencontre dès le Moyen Âge. Voir Duprat A., Les Rois de papier, La caricature d'Henri III à Louis XVI, Paris, Belin, 2002.

41. Pour un aperçu de l'iconographie pro-Assad développée sur Internet depuis la révolte, voir Camus E., "Damas à l'assaut de Facebook ", OWNI, 13 avril 2012. En ligne : (http://owni.fr/2012/04/13/bachar-assad-facebook-propagande-damas/, consulté le 29 juillet 2013). 
ou graffitis qui le qualifient d'âne ou de canard ${ }^{42}$, certains visuels composent des créatures zooanthropiques, greffant une partie de son corps à ces animaux. D'autres le comparent à une girafe, en raison de son long cou, accentuant ce trait physionomique. En suivant la remarque de Philippe Kaenel ${ }^{43}$ rappelant qu'étymologiquement, un «monstre » est ce qui «se montre », on pourrait dire qu'ici, la «monstration du monstre prend valeur de démonstration » du caractère illégitime du président et de ses discours mensongers.

Des visuels s'attaquent également au corps politique du président et à ses représentations officielles. Celui-ci est alors chosifié, désincarné et dépourvu d'humanité. Sur certaines images, son visage se désintègre, à la manière d'un masque qui implose et se désagrège. Réinterprétant la figure hobbesienne du Léviathan, une affiche reconstitue l'effigie souriante du président en train de saluer une foule par le mot «killer », reproduit dans différentes tailles (cf. document 7). Une autre série de visuels subvertit l'imagerie glamour véhiculée par le couple Assad dans la presse locale et internationale. Il apparaît comme totalement insensible aux massacres perpétrés contre la population civile, dont il est désigné comme responsable. Une scène de vie familiale heureuse où l'on voit Asma al Assad appuyée affectueusement sur le dos de son mari pour regarder, avec un de leur fils, des photographies sur l'écran de leur appareil, est incrustée par un effet de montage dans un intérieur luxueux orné de nombreux tableaux. Dans chacun des cadres, ignorés par les protagonistes, absorbés par la contemplation de leur bonheur, ont été apposées les photos d'enfants martyrs. L'image du président, représentée dans l'intimité du cercle familial, est déréalisée dans sa confrontation à un réel dont il est la cause mais auquel il refuse de se confronter. Ce processus de dématérialisation de la figure de pouvoir conduit à sa disparition et sa substitution par des héros ordinaires. Suivant ce procédé, une affiche produite lors d'une campagne officielle lancée en $2005^{44}$ a été détournée. À l'origine, elle représentait le visage souriant du président entouré du drapeau national apposé sur une carte de la Syrie avec l'inscription « Nous t'aimons 45 ». Les couleurs du drapeau ont été inversées et des figures de la révolte apparaissent à la place du visage de Bachar al Assad comme celles de Hamzeh al Khatib, enfant de 13 ans enlevé et tué par les forces de sécurité à Deraa ou d'Abdel Bassit al Sarout, gardien de l'équipe de football nationale, reconverti en meneur de manifestations puis en combat-

42. Selon la rumeur, c'est le nom affectueux qu'utiliserait sa femme dans l'intimité.

43. Kaenel P., "L'apprentissage de la déformation : les procédés de la caricature à la Renaissance ", Sociétés E Représentations, 10, 2000, pp. 81-102.

44. Le régime était alors ostracisé sur la scène internationale, soupçonné d’être impliqué dans l'assassinat de l'ex-Premier ministre libanais Rafiq al-Hariri. Ce contexte avait donné lieu à une surenchère patriotique, orchestrée par des sociétés de communication privées connectées au régime : affiches, banderoles, drapeaux syriens et chants à la gloire du président avaient alors colonisé l'espace public de la capitale. Voir Boëx C., "Produire du sens : les enjeux politiques de l'expression dans l'espace public ", Revue des mondes musulmans et de la Méditerranée, 115-116, 2007, pp. 231-248.

45. Écrite en arabe dialectal syrien [menhabbak] et non en arabe littéraire, afin de mieux souligner le caractère populaire de la déclaration. 
tant à Homs. Les «timbres de la révolution » exposés sur une page Facebook 46 s'arrogent une prérogative étatique pour générer de nouveaux symboles nationaux : protestataires anonymes, opposants syriens célèbres, villes ou évènements emblématiques de la révolte.

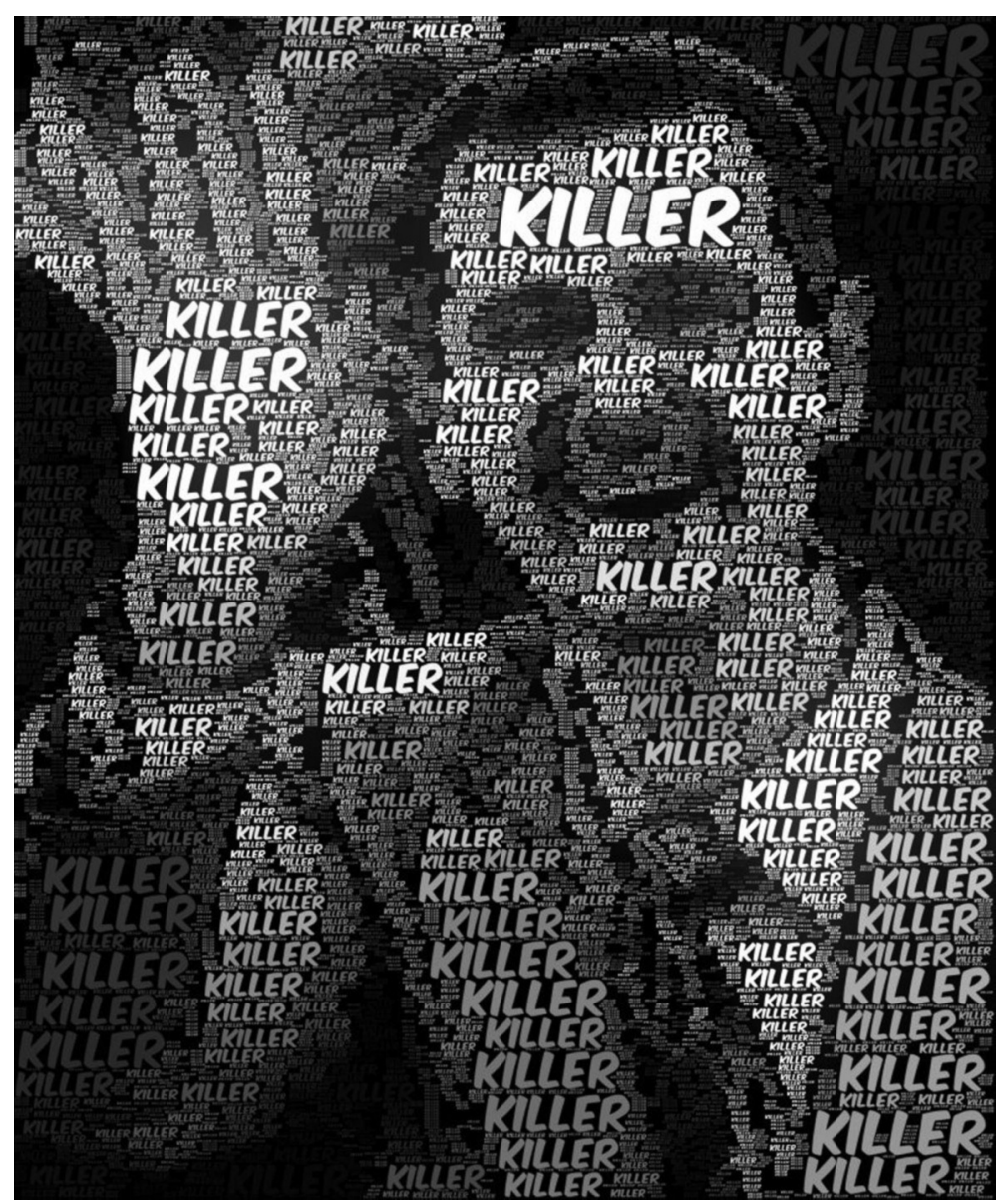

Document 7

46. En ligne : http://www.facebook.com/Stamps.of.the.syrian.revolution (consultée le 29 juillet 2013). 
Ce tour d'horizon de la grammaire iconographique de la révolte, forcément lacunaire, fait néanmoins apparaitre son caractère polymorphe et foisonnant, imprégné par une multitude de styles, de supports et de registres. Ce constat atteste le rôle central de la communication visuelle dans ce contexte singulier, exacerbé par la conjonction de trois facteurs : l'embargo médiatique imposé par le régime, la libération d'une parole muselée pendant plus de quarante ans et l'accessibilité de technologies informatiques et numériques. Mobilisée aussi bien dans la rue que dans l'espace virtuel, l'iconographie constitue un support d'expression politique incontournable. Elle contribue à la coordination des actions protestataires ainsi qu'à leur lisibilité, tout comme elle donne à voir un imaginaire politique en train de s'inventer, modelé pour l'instant, par la négation et l'abolition du précédent. À défaut de faire référence à un programme politique déterminé, dont les bases sont encore loin d'être fixées, la production iconographique est contingente de l'horizon de sens de la révolte : elle relaie ses mots d'ordre, iconise certaines de ses figures individuelles ou collectives et met en relief certains de ces événements marquants. Les thématiques ainsi que les choix des techniques et des supports révèlent par ailleurs la diversité de ses acteurs. Cet éclectisme renvoie également aux multiples espaces au sein desquels cette production iconographique s'élabore. Malgré la fragmentation du territoire due à la répression ${ }^{47}$, qui rend difficile la coordination du mouvement sur le terrain, on observe une stabilisation des formes de l'iconographie ainsi que des récurrences thématiques, même si les styles varient. Les nouvelles technologies de l'image contribuent à ce processus de stabilisation en ce qu'elles permettent un désenclavement et une circulation des techniques et des imaginaires. Elles inscrivent également les dispositifs iconographiques dans l'expérience et la performance protestataire, grâce à la vidéo. Par ailleurs, l'espace de la diffusion et de la création de l'image numérique permet de contourner la répression : d'une part, il préserve la matérialité et la mémoire de la production iconographique élaborée dans la rue et d'autre part, il constitue un espace alternatif de création et de circulation, outrepassant la censure médiatique. L'iconographie ainsi médiatisée par les techniques numériques mobilise différents régimes de visibilité, opérant un va-et-vient entre espaces physiques et virtuels, entre échelles locales, nationales et globales. De multiples points de passage entre différents usages de l'iconographie dans les mobilisations collectives et diverses formes d'activisme en ligne par la vidéo sont ainsi mis au jour. Plus largement, la grammaire iconographique de la révolte en Syrie invite à une réflexion sur les manières dont l'iconographie protestataire se renouvelle et se transforme au contact du numérique, dans sa matérialité ainsi que dans ses espaces de fabrication et de diffusion.

47. Vignal L., "Syrie, anatomie d'une révolution », La vie des idées, 2012, en ligne : http://www.laviedesidees.fr/Syrie-anatomie-d-une-revolution.html (consulté le 29 juillet 2013). 